\title{
EVALUACIÓN DEL GRADO DE AUTORITARISMO Y RIGIDEZ EN EL ESTILO EDUCATIVO DEL DOCENTE*
}

\author{
EVALUATION OF AUTHORITARIANISM AND INFLEXIBILITY \\ IN EDUCATIONAL STYLE OF TEACHER
}

\author{
Francesco Petricone Chiarilli**
}

\section{RESUMEN}

El grado de rigidez y autoritarismo apreciable en el estilo educativo del docente puede ser un obstáculo para el logro de los objetivos educativos. El estudio propone su evaluación a través de un cuestionario aplicable al profesorado que ejerce su función en los centros de educación formal. Para valorar las condiciones técnicas del instrumento construido, el Cuestionario sobre el Estilo Docente (CED) fue aplicado a un grupo de 170 maestros y profesores de la escuela primaria y secundaria. Los resultados dan apoyo a la fiabilidad y validez de constructo para este cuestionario y permiten elaborar algunos perfiles y una tipología según el grado de rigidez y autoritarismo presente en el estilo docente de los profesionales de la educación.

Palabras clave: estilo docente, estilo educativo, cuestionario de rigidez y autocracia.

\begin{abstract}
The degree of inflexibility and authoritarianism in the educational style of the teachers can be a problem for achieving educational objectives. The study proposes its evaluation through an applicable questionnaire to the professorship that exercises their active function in the different formal education centres. To value the technical conditions of the Questionnaire on the Educational Style (CED) it was administered to a group of 170 teachers of the elementary and secondary school. The results give support to the reliability and validity of construct for this questionnaire and permit to elaborate some pro-
\end{abstract}

* Este artículo es un extracto de parte de la tesis doctoral en psicología del autor, titulada: EL Estilo Educativo del Docente (ED) y su Relación con el Estilo Educativo de su Familia de Origen (EFO). Universidad Complutense de Madrid. Facultad de Psicología.

** Doctor en Psicología por la Universidad Complutense de Madrid. Profesor titular jubilado de la Universidad Pedagógica Experimental Libertador (UPEL), Venezuela. Cofundador y director del Centro de Asesoramiento e Investigación Psico-educativa (Caipe). E-mail: fpetric@teleline.es. 
files and typology according to the degree of inflexibility and authoritarianism present in the educational style of the professionals of education.

Key words: educational style, teaching style, questionnaire of inflexibility and authoritarianism.

\section{Introducción}

El concepto de estilo educativo constituye el germen de la pedagogía y la didáctica. La forma cómo se transmiten los contenidos para la preparación de los niños, adolescentes y jóvenes, así como los comentarios valorativos de la información que se dan sobre ellos, tienden a generar aprendizajes distintos según sea el caso (Bennet, 1979; Cthran, Hodges \& Ward, 2000; Joyce y Weil, 1996; Lunemberg \& Korthangen, 2003). Por lo tanto, la conciencia y el conocimiento que se tenga sobre el estilo educativo será un factor importante a tener en cuenta para el logro de los objetivos docentes propuestos en los programas de educación.

Dentro del marco educativo, entre las funciones de la orientación cabe destacar el apoyo y asesoramiento debido a los docentes que se dedican a sus labores de enseñanza. En este sentido, se ha recogido la idea de Sócrates quien decía que la sabiduría no consiste en conocer cosas, sino en el descubrir el modo de conocerlas, con lo cual se hace referencia implícita a la importancia del estilo de educar y enseñar.

El presente artículo pretende aportar, por una parte, la información sobre un instrumento auto-aplicable, sencillo y con suficiente grado de fiabilidad y validez, que pueda ser utilizado por los docentes para valorar el propio estilo educativo predominante en el ejercicio de su profesión: y por otra, describir los resultados de una investigación sobre el estilo educativo docente que permitió construir una tipología del mismo sobre la base del grado de autoritarismo y rigidez manifestada en el mismo.

El esquema tradicional del estilo docente se refería únicamente a la manera de impartir los contenidos académicos por parte de los profesores y la forma en que éstos ejercían su autoridad y liderazgo para imponer la disciplina en clase y motivarlos hacia el aprendizaje. Este concepto ha evolucionado e incorpora otros elementos de tipo personal.

Para ser considerados como tales, los estilos educativos han de reunir por lo menos dos condiciones básicas:

a) mantener cierta consistencia en su continuidad temporal, es decir ser habituales y

b) ser percibidos como una caracterización de la manera de comportarse de la persona que los manifiesta.

Por lo tanto están íntimamente vinculados a las personalidades de quienes ejercen la educación, sean padres, profesores o docentes, instructores o enseñantes. De tal modo que si estos, por ejemplo, tienden a transmitir sus enseñanzas con demostraciones de estilo pesimista o, por el contrario, optimista, el niño o joven percibirá (y probablemente aprenderá) los contenidos expresos de lo que se pretende enseñar, pero también algo sobre el sentimiento de optimismo o pesimismo que los acompaña.

En este sentido, se puede describir a los estilos educativos como modos determinados de las personas para influir y enseñar, caracterizados por formas particulares de comunicar los conocimientos, los sentimientos y las actitudes a través de determinadas expresiones, gestos, palabras y acciones o comportamientos propios del ser humano. 
A objeto de describir una primera clasificación de los estilos educativos docentes como estudio preliminar (Petricone, 2000), fueron encuestados 35 profesores a quienes se les preguntó sobre qué calificativos utilizarían para describir el estilo educativo de los docentes. Los calificativos obtenidos fueron clasificados como se muestra en la tabla 1.

De los tipos de clasificaciones anteriores se optó por la que nos permite explorar el peso que tiene en ella la forma de control de la autoridad, la afectividad, el liderazgo y la comunicación (Baumrind, 1972; Darling y Steinberg, 1993).

Es decir, se asume que quienes ejercen la función educadora pueden hacerlo preponderantemente con uno de los siguientes estilos:

- Rígido y autocrático: ordena, impone, adoctrina, ostenta el poder y la autoridad de manera exclusiva, decide los contenidos y las formas de impartirlos y evaluarlos, mantiene una disciplina férrea, la cual es asumida como un fin en sí misma y sus relaciones afectivas son distantes.

- Negligente y anárquico: deja hacer sin manifestar interés, abandona, muestra indiferencia por las actividades y aprendizajes, no evalúa o lo hace para cumplir con lo estipulado, no manifiesta ni tiene poder o autoridad alguna.

- Democrático y responsabilizante: Expone, cuestiona, estimula y promueve evaluaciones significativas tanto para los alumnos como para sí mismo, da y recibe confianza manteniendo el respeto mutuo y los niveles de autoridad, discute, ofrece y recibe retro-información, da alternativas de responsabilidad entre todos, utiliza el poder para resolver problemas u obtener los beneficios acordados entre todos, la disciplina es asumida como un medio y no como un fin, permite la manifestación de sentimientos y afecto dando muestras de ello.

Conscientes de que las clasificaciones sólo son un recurso teórico de aprovechamiento práctico y que la amplitud de las posibles clasificaciones de estilo educativo docente que podrían deducirse de sus combinaciones es bastante extenso (puesto que no existen tipologías absolutamente puras), hemos optado por la categorización del grado de autoritarismo y rigidez existente en la manifestación del estilo educativo del docente, dada la connotación peyorativa que envuelve este componente vinculada a sus consecuencias indeseables en la educación y a su obstaculización para el cambio y progreso en el devenir humano (Cevallos y Rodrigo, 1998; Hoffman, 1970; Lamborn, Mounts, Steinberg y Dornbusch, 1991).

Así se optó por la construcción de un instrumento destinado a la valoración del grado de rigidez y autoritarismo en el estilo educativo del docente. Es decir, el Cuestionario sobre el Estilo Educativo del Docente, subtitulado CED con objetivos claros y específicos. A saber:

1. Permitir identificar el estilo educativo predominante y propio del docente que desarrolla sus funciones profesionales como educador, sobre la base hipotética de que dichos estilos están caracterizados por comportamientos, actitudes y pensamientos que pueden ser identificados, agrupados y categorizados a través de un instrumento elaborado con tales fines.

2. Disponer de un instrumento que permita elaborar ciertas tipologías sobre el estilo educativo de los docentes en términos de grado de autoritarismo y rigidez presente en cada tipo. 
TABLA 1: Clasificación de estilos educativos (F. Petricone, 2000).

\begin{tabular}{|c|c|}
\hline Criterio & Categoría \\
\hline Centrado en el tiempo: & $\begin{array}{l}\text { Acelerado } \\
\text { Estancado } \\
\text { Pausado } \\
\text { Acompasado }\end{array}$ \\
\hline Centrado en el sujeto & $\begin{array}{l}\text { Crítico } \\
\text { Nutriente } \\
\text { Sobre-protector } \\
\text { Indiferente } \\
\text { Hiper-exigente }\end{array}$ \\
\hline Centrado en el programa & $\begin{array}{l}\text { Rígido y directivo } \\
\text { Indiferente } \\
\text { Adaptado } \\
\text { Flexible } \\
\text { Creativo }\end{array}$ \\
\hline Centrado en el ambiente & $\begin{array}{l}\text { Disciplinario y normativo } \\
\text { Anarquista y "dejar hacer" } \\
\text { Ambientalista y estimulante }\end{array}$ \\
\hline Centrado en la comunicación (Satir, 1972) & $\begin{array}{l}\text { "aplacador" } \\
\text { "acusador" } \\
\text { "superrazonable" } \\
\text { "irrelevante" }\end{array}$ \\
\hline Centrado en el contexto & $\begin{array}{l}\text { Individualista } \\
\text { De equipo } \\
\text { Sistémico } \\
\text { Comunitario }\end{array}$ \\
\hline Centrado en los ideales & $\begin{array}{l}\text { Religioso } \\
\text { Político } \\
\text { Social } \\
\text { Cultural }\end{array}$ \\
\hline Centrado en el enfoque didáctico & $\begin{array}{l}\text { Mayéuticos } \\
\text { Objetivistas } \\
\text { Empiristas } \\
\text { Experimentalista } \\
\text { Experiencialistas } \\
\text { Fenomenólogos } \\
\text { Guestaltistas }\end{array}$ \\
\hline $\begin{array}{l}\text { Centrado en el control de la autoridad y el liderazgo } \\
\text { (Baumrind, 1972) }\end{array}$ & $\begin{array}{l}\text { Rígido y autocrático } \\
\text { Negligente y anárquico } \\
\text { Democrático y responsabilizante }\end{array}$ \\
\hline Centrado en su funcionalidad & $\begin{array}{l}\text { Funcional y participativo } \\
\text { Disfuncional e impositivo }\end{array}$ \\
\hline
\end{tabular}




\section{Método}

Se procedió a la elaboración de un diseño para la selección de la muestra (el cual no fue posible ejecutar debido a la poca accesibilidad a los docentes en los respectivos colegios o instituciones educativas escogidas al azar. Seguidamente se visitaron los centros docentes para obtener la autorización respectiva a la administración de los instrumentos recaudadores de los datos pertinentes al estudio. La entrevista con los directores estuvo acompañada de un material escrito explicativo de los objetivos de la aplicación de los instrumentos y una copia de los mismos.

Obtenido el permiso, se entregaron a los profesores (previa manifestación de su disposición a cooperar con el estudio) los sobres abiertos contentivos del instrumento, y se les solicitó devolverlos debidamente cumplimentados en el sobre cerrado (anonimato y garantía de privacidad de los datos aportados) el día previamente acordado. Finalmente se ha se trabajado con la información aportada por un total de 170 instrumentos debidamente cumplimentados.

Los datos fueron procesados utilizando el paquete estadístico SPSS versión 10.0 para Windows, a excepción del análisis de cluster último en que se empleó una modalidad mixta del programa estadístico computarizado: Syestemmè pour l'analyse des dones (SPAD). La unidad procesadora del análisis fue el Área de Informática y Comunicaciones de la Universidad Complutense de Madrid (atención de D. Ricardo García Mata).

El procedimiento general seguido se sintetiza en los siguientes pasos:

En primer lugar se realizó un análisis exploratorio destinado a obtener una descripción más detallada, depurada y precisa de las variables en estudio. Éstas, inicialmente, fueron objeto de consideración en forma independiente (variable por variable) y luego combinadas entre sí por medio de una serie de enlaces (análisis de tablas de contingencia). Algunas variables fueron dicotomizadas o transformadas a un mismo tipo de escala, según fuera necesario, para luego ser sometidas a un análisis factorial y a un análisis de conglomerados. Por último, con los resultados obtenidos, se elaboraron varios perfiles de utilidad tipológica.

\section{Descripción del instrumento: Cuestionario sobre Estilo Docente (CED)}

Este cuestionario fue diseñado para obtener una puntuación sobre el grado de Rigidez y Autocracia presentes en el Estilo educativo del Docente. Se elaboraron 41 ítems con una escala tipo Likert, que debían ser respondidos de acuerdo con una valoración que va del 1 ("Nunca") al 5 ("Siempre"), pasando por los puntos intermedios (2 = "a veces", 3 = "ocasionalmente" y 4 = "casi siempre"). Las puntuaciones finales se situaron en un continuo cuyas categorías polarizadas indican un componente muy elevado o muy bajo de "rigidez y autocracia" en el estilo educativo docente. Del total de ítems sólo 15 obtuvieron unos índices altos de validez y fiabilidad (método de juicio de expertos y análisis de items).

La valoración final corresponde a la totalización de puntos en la escala. A mayor puntuación, más tendencia a un estilo educativo rígido y autocrático. Para el computo total fueron invertidos (por sus características) los valores de algunos ítems. Previo a la presentación de los ítems del cuestionario, el docente debía contestar una serie de preguntas destinadas a recoger la información relevante para el estudio, así como escoger tres términos calificativos de la percepción del propio estilo docente. 


\section{Participantes en el estudio}

Todos los participantes son "profesores en ejercicio" de alguno de los centros docentes católicos de la ciudad de Madrid. La edad de los mismos va desde los 23 hasta los 74 años. El promedio de edad del grupo es de 42 años. En cuanto al estado civil, un 8,8\% no dio respuesta a su condición, pero más de la mitad $(52,4 \%)$ respondió ser soltero(a) lo que, unido a quienes son religiosos(as) $(1,8 \%)$, hace un porcentaje total que asciende a un $54,2 \%$. El $32,4 \%$ responde estar casado(a) y el $4,8 \%$ de las personas encuestadas (que estuvieron o están casadas) confiesan haber tenido problemas con su pareja lo que ha culminado en la separación o el divorcio. El $76 \%$ del profesorado que participó en el estudio son mujeres y el 23,5\% hombres.

Respecto a la credencial que ostenta el profesorado, aproximadamente el 43,5\% ha obtenido el magisterio (estudios superiores con tres años de duración), mientras que el 52,9\% tiene la licenciatura y casi el $1,8 \%$ el doctorado. Hay un pequeño porcentaje $(1,2 \%)$ que ejerce la docencia habiendo obtenido un título distinto al de profesor o docente propiamente dicho.

Sobre el tiempo de experiencia acumulado en el ejercicio como docentes, la gama va desde un año como mínimo hasta más de cincuenta. Se aprecia una leve predominancia en la frecuencia de docentes con menos de 18 años de experiencia $(58,2 \%)$ con respecto al $41,8 \%$ que la superan. Más del $20 \%$ del total de los docentes han traspasado los 28 años de ejercicio en sus labores y más del 5\% los 38 años.

Los ciclos educativos donde dictan clases los docentes encuestados se distribuyen prácticamente en partes iguales: el $43,5 \%$ en primaria e infantil y $42,9 \%$ en secundaria (ESO y Bachiller). Casi un 14\% lo hacen en ambos ciclos.

\section{Resultados}

Auto-calificativo del estilo docente (AUTEDO) fue explorado a través de la pregunta: Si tuviera que ponerse algunas de las siguientes etiquetas, ¿cuáles cree Ud. que se ajustarían más a su estilo docente?

Los resultados indican que los docentes, entre una serie de auto-calificativos del propio estilo docente, tienden a escoger mayormente las opciones siguientes (en orden de frecuencia y porcentaje): "flexible y adaptado" (27\%), "creativo" (20\%), "directivo y exigente" (15\%), "crítico" (13\%), "nutriente" (8\%), "acelerado" (6\%) o "pausado" (5\%), "acompasado" (3\%) y "rígido" (3\%).

La consideración de la forma en que aparecen ordenados estos calificativos (de mayor a menor frecuencia) permite afirmar que tanto las respuestas relacionadas con la creatividad como las vinculadas a la flexibilidad y adaptabilidad están entre las más escogidas; las relacionadas con estilos directivos, exigentes y críticos ocupan el tercero y cuarto lugar de frecuencias. Acelerado, pausado, acompasado y rígido están ubicados en esta misma secuencia desde el quinto al último puesto.

Parece existir cierta asociación significativa $\left(X^{2}(9)_{n}=20,678, p<0,05\right)$ entre el autocalificativo con el que identifican los docentes el estilo educativo que suelen utilizar en sus actividades y los años que tienen de experiencia docente. De hecho, los docentes cuya 
experiencia está entre los 3 y 7 años suelen responder positivamente al auto-calificativo de estilo creativo, mientras que a partir de los 38 años en adelante esa relación se invierte (negativa).

De igual manera, los datos de los auto-calificativos de directivo y exigente en cuanto al propio estilo docente según los grupos de tiempo de experiencia, muestran que los docentes más jóvenes o con menos años de experiencia docente (desde uno a 17 años) suelen no calificar su estilo como directivo y exigente. Por el contrario, pasados los 27 años de experiencia, se comienza a diferenciar una asociación positiva con este tipo de elección de estilo educativo.

\section{Fiabilidad y validez de constructo del CED}

De acuerdo con la escala previamente descrita, la valoración final corresponde a la totalización de puntos de dicha escala. A mayor puntuación, más tendencia a manifestar un estilo educativo rígido y autocrático.

Se administró una primera versión del Cuestionario sobre el Estilo Educativo Docente a un grupo de profesores de Madrid en ejercicio y, además de cumplimentar el cuestionario, se les solicitó manifestar su opinión y comentarios u observaciones sobre el instrumento. Al final, las características de las afirmaciones componentes de los ítems sugerían la presencia de tres factores subyacentes al constructo estilo docente:

- Actitud general en el estilo educativo del docente,

- Rasgos de rigidez y autoritarismo en dicho estilo, y

- Actitud laboral en el ejercicio docente

Para su corroboración (validez de constructo), los datos obtenidos fueron sometidos a un análisis factorial cuyos resultados se confrontaron con los inicialmente planteados en forma teórica.

Los resultados dieron apoyo a los planteamientos teóricos sobre los que se elaboró el instrumento y, al final, el cuestionario quedó integrado por 15 ítems y cuatro factores principales. El método utilizado fue el de extracción de componentes principales y la rotación Varimax. Su adecuación para estos datos queda reflejada en los valores de los estadísticos pertinentes, donde se aprecia que la medida de adecuación muestral de Kaiser-Meyer-Olkin alcanza un p-valor de 0,757 , lo cual es considerado "mediano" según la escala de Kaiser (en García Jiménez, Gil Flores y Rodríguez Gómez, 2000) y el Chi-cuadrado de la prueba de esfericidad de Bartlett resultó significativo a nivel de 0,001 .

Establecido el número de cuatro factores y hecha la extracción, los valores de las comunalidades quedaron registradas en la tabla 2 (ver anexos).

Los cuatro factores encontrados explican el $53,55 \%$ de la varianza total y el autovalor (varianza correspondiente) para el $1^{\text {er }}$ factor es de $25,76 \%$, para el segundo es de un $11,53 \%$, para el tercero corresponde un $8,68 \%$ y para el cuarto el $7,58 \%$.

Los factores fueron calificados como sigue:

1) Autoritarismo (variables $\mathrm{N}^{\circ} 19,29,3133$ y 41).

2) Rigidez (variables $N^{\circ} 34,36,38$ y 39). 
TABLA 2: Valores de las comunalidades items del CED.

\begin{tabular}{|c|c|c|}
\hline Variables & Inicial & Extracción \\
\hline item 2: Evito corregir ...para no tener problemas & 1 & 0,687 \\
\hline $\begin{array}{l}\text { item 3: No tengo vocación docente... me veo obligado(a) por motivos } \\
\text { económicos }\end{array}$ & 1 & 0,518 \\
\hline item 10: Temo que... no me respeten y por eso ejerzo mucho control & 1 & 0,440 \\
\hline item 17: Siento soledad y amargura cuando estoy en clase & 1 & 0,462 \\
\hline $\begin{array}{l}\text { item 18: Estoy pendiente de la hora que termina la clase... para hacer } \\
\text { otras cosas... }\end{array}$ & 1 & 0,560 \\
\hline $\begin{array}{l}\text { item 19: Los alumnos(as) deben aprender que el docente es quien manda } \\
\text { en clase }\end{array}$ & 1 & 0,641 \\
\hline $\begin{array}{l}\text { item 23: Existen alumnos(as) que tienen la facultad de hacerme perder el } \\
\text { control... }\end{array}$ & 1 & 0,528 \\
\hline item 29: A los estudiantes hay que "ponerlos en su sitio" para que... & 1 & 0,588 \\
\hline item 31: El castigo es el mejor método para corregir... & 1 & 0,564 \\
\hline item 33: Vale ser más autócrata que demócrata & 1 & 0,303 \\
\hline $\begin{array}{l}\text { item 34: No me gusta como soy con mis estudiantes, pero no puedo } \\
\text { remediarlo }\end{array}$ & 1 & 0,601 \\
\hline $\begin{array}{l}\text { item 36: Me resulta difícil dar manifestaciones de afecto a mis } \\
\text { alumnos(as) }\end{array}$ & 1 & 0,651 \\
\hline $\begin{array}{l}\text { item 38: No acepto que mis alumnos(as) me hagan sugerencias sobre } \\
\text { como dar una clase }\end{array}$ & 1 & 0,483 \\
\hline $\begin{array}{l}\text { item 39: Siento una preferencia especial por los que obtienen buen } \\
\text { rendimiento }\end{array}$ & 1 & 0,465 \\
\hline item 41: La mayoría ...necesita ser & 1 & 0,541 \\
\hline
\end{tabular}

Método de extracción: Análisis de Componentes Principales.

3) Insatisfacción profesional (variables $N^{\circ} 3,17,18$ y 23) y

4) Defensividad (variables $N^{\circ} 2$ y 10) (tabla 3 ).

Como estrategia alternativa a la anterior, e igualmente aplicable a este caso, se empleó el análisis de conglomerados y se encontraron las mismas agrupaciones indicadas como factores en el análisis factorial. El dendrograma resultante reveló que la mayoría de las variables se unían a distancias no tan próximas como se esperaba, pero lo suficiente para mostrar cierta homogeneidad entre ellas. Los conglomerados finales fueron asumidos a partir de las líneas horizontales que los representan como tales en las puntuaciones que van de 13 a 15 en la escala de distancias. Así, quedaron constituidos cuatro conglomerados medianamente homogéneos. 
TABLA 3: Matriz de componentes rotados.

\begin{tabular}{|c|c|c|c|c|}
\hline \multirow{2}{*}{ Variables } & \multicolumn{4}{|c|}{ Componente } \\
\hline & 1 & 2 & 3 & 4 \\
\hline $\begin{array}{l}\text { item 19: Los alumnos(as) deben aprender que el docente es quien } \\
\text { manda en clase }\end{array}$ & ,781 & & & \\
\hline item 29: A los estudiantes hay que "ponerlos en su sitio" para que... & 686 & ,313 & & \\
\hline item 31: El castigo es el mejor método para corregir... & ,608 & & & ,440 \\
\hline $\begin{array}{l}\text { item 41: La mayoría ...necesita ser presionado(a) para que } \\
\text { aprendan... }\end{array}$ &, 591 & & & ,367 \\
\hline item 33: Vale ser más autócrata que demócrata &, 510 & & & \\
\hline $\begin{array}{l}\text { item 36: Me resulta difícil dar manifestaciones de afecto a mis } \\
\text { alumnos(as) }\end{array}$ & & ,780 & & \\
\hline $\begin{array}{l}\text { item 34: No me gusta como soy con mis estudiantes, pero no puedo } \\
\text { remediarlo }\end{array}$ & & ,684 & ,351 & \\
\hline $\begin{array}{l}\text { item 39: Siento una preferencia especial por los que obtienen buen } \\
\text { rendimiento }\end{array}$ & & ,597 & & \\
\hline $\begin{array}{l}\text { item 38: No acepto que mis alumnos(as) me hagan sugerencias } \\
\text { sobre como dar una clase }\end{array}$ & & ,556 & & ,316 \\
\hline $\begin{array}{l}\text { item 23: Existen alumnos(as) que tienen la facultad de hacerme } \\
\text { perder el control... }\end{array}$ & & & ,699 & \\
\hline $\begin{array}{l}\text { item 18: Estoy pendiente de la hora que termina la clase... para } \\
\text { hacer otras cosas... }\end{array}$ & & & ,688 & \\
\hline $\begin{array}{l}\text { item 3: No tengo vocación docente... me veo obligado(a) por } \\
\text { motivos económicos }\end{array}$ & & & ,639 & \\
\hline item 17: Siento soledad y amargura cuando estoy en clase & & & ,565 & \\
\hline item 2: Evito corregir ...para no tener problemas & & & & ,794 \\
\hline item 10: Temo que... no me respeten y por eso ejerzo mucho & & ,328 & &, 550 \\
\hline
\end{tabular}

Utilizando la construcción teórica como fuente para ser confrontada con estos resultados, se obtuvo un soporte empírico favorablemente satisfactorio ante el criterio de validez de constructo para el Cuestionario sobre el Estilo Docente (CED).

El coeficiente de consistencia interna de este instrumento, alcanzó un valor alpha de Crombach aproximado de 0,79 lo que, teniendo presente el número de ítems que lo componen (15 ítems), indica un adecuado índice de fiabilidad.

Los valores de la escala en términos categorizados por niveles y sus respectivas frecuencias para el grupo de docentes en estudio refleja que el $16,1 \%$ de los encuestados resultó expresar un alto grado de rigidez y autocracia en su estilo docente; sólo el 1,2\% manifestó escasa o poca rigidez y autocracia en dicho estilo y más del $80 \%$ es mediana- 
mente $(39,3 \%)$ o tiene tendencia a ser rígido y autocrático en la forma de ejercer la docencia $(43,5 \%)$.

Estos resultados fueron relacionados con el grupo de variables demográficas (edad, sexo, estado civil, años de experiencia laboral) y familiares (número de miembros familiares, lugar de nacimiento, educación y profesión de los padres) de los docentes y sólo algunas mostraron estar estadísticamente asociadas con los grados de rigidez y autoritarismo encontrados en el CED. No se reportan aquí estos datos porque deberán ser objeto de otro estudio Sin embargo, a título de ejemplo, resultó significativa la relación existente entre la confirmación de estar ejerciendo la vocación acertada de la docencia y el estilo docente que manifiestan los profesores en dicho ejercicio. Los profesores que ratifican su vocación están asociados directamente con un estilo educativo docente medianamente rígido y autocrático, e inversamente con un estilo altamente rígido y autocrático. Por el contrario, los que reconocen no tener vocación docente, o no están seguros de ella, parecen manifestar un estilo educativo altamente rígido y autocrático.

\section{Perfiles referentes al estilo educativo docente}

Las variables vinculadas a la valoración del estilo docente, después de su descripción y depuramiento, fueron modificadas en su categorización de acuerdo con las siguientes criterios: se asignó el número 1 a las opciones "nunca u ocasionalmente" y el número 2 a las respuestas dadas como "regularmente o casi siempre". Siguiendo los pasos correspondientes, se realizó un corte que permitió conservar tres clusters que agrupan los participantes en tres clases o tipos de estilos docentes:

a) "flexible y no autocrático",

b) "tendiente a la rigidez y autocracia" y

c) "rígido y autocrático".

Las características del grupo catalogado como ejecutor de un tipo de estilo docente no rígido ni autocrático (ver anexo, tabla 4) respondió estar seguro de su vocación docente (97,64\% dentro de la clase o grupo 1) y no es partidario de presionar e imponerse a sus alumnos para que lo respeten. Corrige a los alumnos cuando es necesario, pero no cree que el castigo sea el mejor método para hacerlo. Por lo general dicen no tener dificultad para manifestar su afecto a los alumnos y no suelen tener preferencias por su rendimiento académico. Acepta que sus estudiantes le hagan sugerencias sobre la clase y piensa que vale ser más demócrata que autócrata. Gusta de cómo es y está centrado en sus actividades docentes sin estar excesivamente pendiente de la hora en que concluyen sus clases.

Otro grupo presentó características bastante parecidas a las del anterior, pero con una alta presencia de respuestas de modalidad tipo "no responde". Es decir que posiblemente, ante la duda o la no aceptación de su realidad, a pesar de disponer inicialmente de cinco alternativas, decide no responder como una forma evasiva de la situación. El estilo docente de este grupo se ha calificado como "tendiente a la rigidez y autocracia". Los participantes de este tipo de estilo docente muestran en sus respuestas ciertas dudas sobre su vocación y parecen estar a la defensiva con respecto a sus alumnos ante los cuales suelen sentirse amenazados o vulnerables, por eso tienden a perder sus estribos ante algunos de ellos. 


\begin{tabular}{|c|c|c|c|c|c|c|c|c|c|c|c|c|c|}
\hline ș & 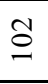 & $\stackrel{?}{ \pm}$ & 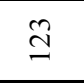 & $\stackrel{\text { I }}{工}$ & ปิ & $\stackrel{\infty}{\stackrel{n}{n}}$ & 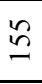 & in & $\stackrel{\varrho}{=}$ & ભે & $\hat{n}$ & $\stackrel{\circ}{ \pm}$ & 气ิ \\
\hline 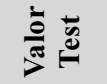 & $\overbrace{6}^{n}$ & $\begin{array}{l}\infty \\
\text { N } \\
0\end{array}$ & D & $\frac{6}{n}$ & $\begin{array}{l}\mathscr{\infty} \\
\infty \\
+\end{array}$ & $\underset{\sim}{\stackrel{\sim}{*}}$ & ले & $\dot{\infty}$ & $\bar{\sigma}$ & $\stackrel{+}{m}$ & $\begin{array}{l}\infty \\
\infty \\
i\end{array}$ & $\begin{array}{l}\text { ָे } \\
\text { i }\end{array}$ & \begin{tabular}{l}
$n$ \\
\multirow{r}{*}{} \\
$i$
\end{tabular} \\
\hline 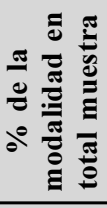 & $\vec{i}$ & $\frac{N}{\infty}$ & $\vec{\sim}$ & $\begin{array}{l}n \\
\tilde{\infty} \\
\dot{\infty}\end{array}$ & $\stackrel{2}{2}$ & $\begin{array}{l}\tilde{2} \\
\dot{d}\end{array}$ & $\begin{array}{l}\text { v } \\
\text { ป̂ } \\
\text { a }\end{array}$ & $\hat{m}^{n}$ & $\begin{array}{l}\tilde{o} \\
\hat{\sigma}\end{array}$ & $\begin{array}{l}\text { I } \\
\text { i }\end{array}$ & $\frac{\hat{a}}{a}$ & ले & $\begin{array}{l}\text { Jे } \\
\dot{a}\end{array}$ \\
\hline 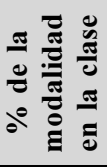 & \begin{tabular}{l}
$\triangleright$ \\
\multirow{+}{*}{} \\
\end{tabular} & $\begin{array}{l}0 \\
0 \\
0\end{array}$ & $\begin{array}{l}\hat{\infty} \\
\infty \\
\infty\end{array}$ & $\frac{r}{2}$ & $\begin{array}{l}\bar{\sigma} \\
\infty \\
\infty\end{array}$ & $\begin{array}{l}\bar{\pi} \\
\text { aे }\end{array}$ & $\begin{array}{l}\text { J } \\
\hat{a}\end{array}$ & $\frac{\Re}{F}$ & $\frac{1}{\approx}$ & $\begin{array}{l}\infty \\
\curvearrowright \\
\infty \\
\infty\end{array}$ & \begin{tabular}{l}
$\infty$ \\
\multirow{2}{n}{} \\
2
\end{tabular} & $\frac{\infty}{\infty}$ & $\begin{array}{l}\text { ț } \\
\hat{a}\end{array}$ \\
\hline 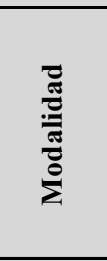 & 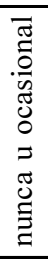 & 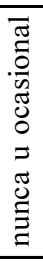 & 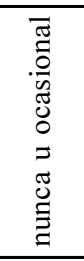 & 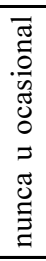 & 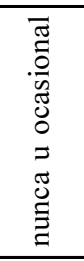 & 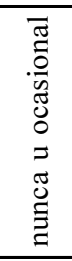 & $\bar{n}$ & 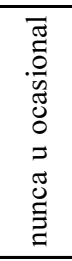 & 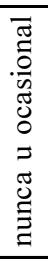 & 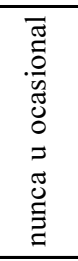 & 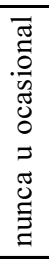 & 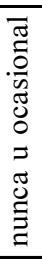 & 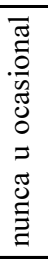 \\
\hline 竞 & 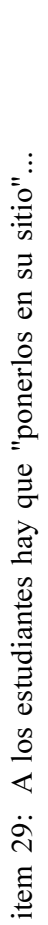 & 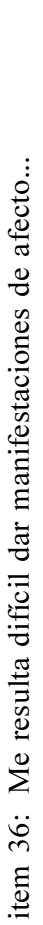 & 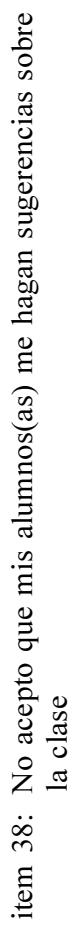 & 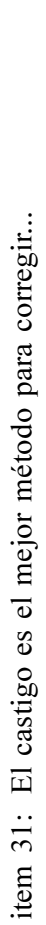 & 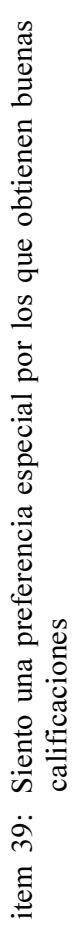 & 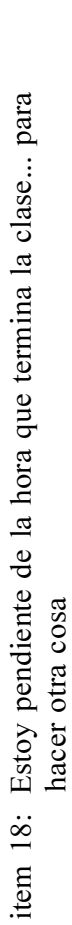 & 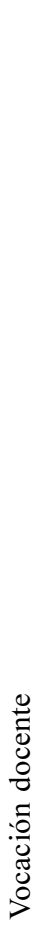 & 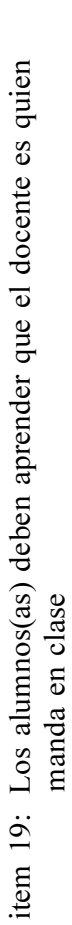 & 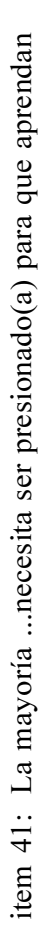 & 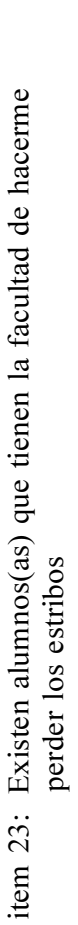 & 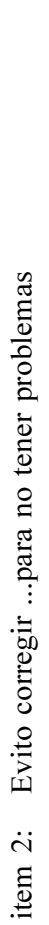 & 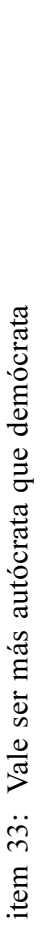 & 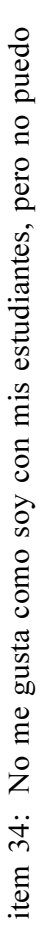 \\
\hline
\end{tabular}


La tercera clase o grupo proporciona respuestas más abiertas y concretas a las afirmaciones. Aun cuando se observan algunas respuestas de tipo "no contesta", éstas parecen deberse más al no reconocimiento o aceptación que a la duda, puesto que se contradicen cuando las afirmaciones involucran una categorización como, por ejemplo cuando se hace referencia expresa a la autocracia. Para este grupo el estilo docente supone que el castigo es el mejor método para corregir a los alumnos, que la mayoría de éstos necesitan ser presionados para que aprendan y es necesario que el docente deje bien claro quien es el que manda en la clase. Tienden a mostrar una preferencia especial por los alumnos que obtienen mejor rendimiento académico. Pero además parece no aceptar que se les califique de rígidos y autócratas.

\section{Conclusiones}

En lo relativo al su ejercicio profesional, los términos escogidos por los docentes sobre el propio estilo educativo fueron, en orden de frecuencia, los siguientes: "flexible y adaptado" (27\%), "creativo" (20\%), "directivo y exigente" (15\%), "crítico" (13\%) o "nutriente" (8\%), "acelerado" (6\%), ), "pausado" (5\%), "acompasado" (3\%) y "rígido" (3\%). Lo que indica que la mayoría de los docentes tienden a calificar de forma positiva su estilo docente. Cabe preguntarse cuánto puede tener que ver esta elección con lo sugerido por Alberdi (1995) sobre la tendencia del español a evitar calificarse como "autoritario".

Un análisis más detallado nos lleva a observar que estos calificativos tiene varias vertientes, por lo que se puede ser "creativo y crítico" "creativo y directivo", "creativo y nutriente", "creativo acelerado" o "creativo pausado" e incluso "creativo flexible". Igualmente se puede ser de estilo "tolerante y acelerado o pausado", "directivo, exigente, creativo y nutriente". Es decir que las formas puras no existen en la realidad, sino que, en nuestro caso, responden a una predominancia reconocida de un estilo por parte de los participantes en el estudio.

Aunque los datos expuestos no son determinantes, se observa que los docentes cuya experiencia es menor tienen más tendencia a calificarse con un estilo educativo creativo, flexible y adaptado que los de experiencias más largas. Lo que podría llevar a suponer que, si han sido creativos alguna vez, han dejado de serlo a medida que aumentan su experiencia. El caso contrario sucede con el calificativo de directivo y exigente que vienen a ser más utilizados por los que superan los 28 años de experiencia en su trabajo. Pero también podría tener otra explicación si se tiene en cuenta el tipo de formación profesional que han recibido.

En cuanto a la aplicación del Cuestionario sobre el Estilo Docente para obtener un índice del grado de rigidez y autocracia del estilo educativo docente (ED) a través de una escala construida sobre el grado de defensividad, la rigidez y la actitud laboral, el estudio de los factores de validez y fiabilidad fueron satisfactorios y permitieron diferenciar tres tipos de estilos educativos del docente:

- El tipo 1: estilo docente flexible y democrático. Este estilo es el practicado por los profesionales con vocación docente que consideran innecesarias las imposiciones y las presiones para obtener el respeto y aprecio de sus alumnos; por lo tanto, no creen 
que el castigo sea la mejor forma de corregirlos cuando es necesario hacerlo. Suelen aceptar que los estudiantes les hagan sugerencias sobre sus clases y defienden por convicción la idea de ser predominantemente democráticos. Son capaces de expresar abiertamente su afecto hacia los alumnos y no condicionan sus preferencias al rendimiento académico de éstos. Manifiestan estar a gusto con su manera de ser y con las actividades desarrolladas en clase con sus alumnos.

- El tipo 2: estilo docente tendiente a la rigidez y autocracia. Se trata de un estilo docente que no es flexible y democrático, pero, si bien se inclina a ser rígido y autocrático, tampoco puede calificarse como tal. Se caracteriza por presentar algunas de las formas que se describen a continuación en el tercer tipo, pero además es ejercido de forma temerosa, es decir sin o poca autoridad, siendo más una forma reactiva de defensa que el ejercicio de un estilo educativo por convicción.

- El tipo 3: estilo docente rígido y autocrático. Al contrario del caso anterior, este estilo educativo se pone en práctica por quienes están convencidos de que es así como debe hacerse la educación y socialización. Se justifica el uso del castigo y la represión como mejor método para corregir a los alumnos, así como la presión para el logro del aprendizaje. Se mantiene la figura de autoridad del docente basada en el poder manifiesto en todo momento, por lo que no se aceptan ni permiten las sugerencias del estudiantado. Las preferencias entre los estudiantes están regidas por el rendimiento académico y la disciplina mantenida.

Así mismo, los resultados indican que el 16\% de los profesionales participantes en el estudio manifiestan tener un "estilo alto en rigidez y autocracia", el 39,3\% obtienen puntuaciones indicativas de un estilo "medianamente rígido y autocrático", el 43,5 tiende a la rigidez y autocracia y el $1,2 \%$ obtienen un "índice de rigidez y autocracia escaso".

Parece ser que la "vocación" es una variable significativamente relacionada con el índice de rigidez y autocracia en el estilo docente. De hecho, los participantes que ratifican su vocación están asociados directamente con un estilo educativo docente escasamente rígido y autocrático, e inversamente con un estilo altamente rígido y autocrático. Por el contrario, los docentes que reconocen no tener vocación docente, o no están seguros de ella, parecen manifestar una tendencia hacia el estilo educativo altamente rígido y autocrático.

\section{Limitaciones del estudio}

Las principales limitaciones del estudio se relacionan con el grado de generalización de los datos. Ante la duda sobre la validez externa de los resultados aquí encontrados (por cuanto no se pudo cumplir con las pautas de escogencia de una muestra que garantizan la representatividad muestral de un universo determinado del profesorado de los centros docentes de Madrid), cabe tener presente algunos argumentos en su favor:

1) el número de los participantes satisface suficientemente el criterio del tamaño de una muestra para una población finita;

2) la población a la que se refiere es bastante homogénea (profesores o docentes de los centros católicos ubicados en la ciudad de Madrid) y, consecuentemente con muchas 
características de varianza bastante baja, por lo tanto, al requerir un menor número de participantes, puede considerarse un modelo reducido satisfactorio del universo que pretende representar;

\section{Implicaciones para la orientación}

La utilización de estos resultados con fines de orientación puede abarcar, en principio, dos grandes áreas.

La primera tiene que ver con la atención directa de los docentes quienes con frecuencia se ven bloqueados ante las distintas situaciones conflictivas que se le presentan en su labor diaria de la enseñanza. Por ejemplo, nuestra experiencia de más de treinta años de trabajo con docentes nos permite exponer que en ocasiones éstos ven nuestra función orientadora como una intromisión en su trabajo, sobre todo cuando se les insinúa que, ante un conflicto, posiblemente su estilo educativo sea el que esté obstaculizando su resolución. Quizás el resultado de la auto-administración del CED llegue a ayudar o concienciar al docente o al profesor sobre ello, o retro-informar al orientador sobre lo acertado o equivocado de una hipótesis que necesita verificar.

La segunda puede ser una contribución informativa para el perfil deseable en la formación profesional del profesorado y los educadores en general. En este sentido, los curricula debieran incorporar en la formación profesional específica los contenidos referentes a los estilos educativos.

Para concluir, es importante tener presente que este estudio constituye sólo uno de los varios aspectos tratados en una investigación más amplia cuyo objetivo fue describir la posible asociación existente entre el estilo educativo docente y el estilo educativo predominante en su familia de origen.

\section{Referencias bibliográficas}

Alberdi, J. (1995). Informe sobre la situación de la familia en España. Madrid: Ministerio de Asuntos Sociales.

Baumrind, D. (1971). "Current patterns of parental authority". Developmental Psychology Monographs, 4 (1), 1-102.

Bennet, H. (1979). Estilos de enseñanza y progreso de los alumnos. Madrid: Ed. Morata.

Cevallos, E. y Rodrigo, M. J. (1998). "Las metas y estrategias de socialización entre padres e hijos". En M. J. Rodrigo y J. Palacios (Eds.), Familia y desarrollo humano (pp. 225-243). Madrid. Alianza.

Cthran, D.; Hodges K., P y Ward, E. (2000). "Students' experiences with and perceptions of teaching styles". The Journal of Research and Development in Education. Vol. 33, pp. 93-103.

Darling, N. \& Steinberg, L. (1993). "Parenting Style as Context: An Integrative Model". Psychological Bulletin, 113, pp. 487-496.

Hoffman, M. L. (1970). "Conscience, personality and socialization techniques". Human Development, 13, pp. $90-126$.

Joyce, B. R. \& Weil, M. (1996). Models of Teaching. Boston, MA: Allyn \& Bacon. 
Lunemberg, M. \& Korthangen, F. A. J. (2003). “Teacher educators and student-directed learning”. Teaching and Teacher Education. 19, pp. 29-44.

Lamborn, S. D., Mounts, N. S., Steimberg, L. \& Dornbusch, S. M. (1991): "Patterns of competence and adjustment among adolescents from authoritative, authoritarian, indulgent and neglectful families", en Child Development, 62, pp. 1049-1065.

Musitu, G. y Allatt, P. (1994). Psicosociología de la familia. Valencia: Editorial Albatros.

Petricone, F.(2000). Exploración del estilo educativo de dos grupos de docente: uno de Maracay (Venezuela) y otro de Madrid (España). Estudio piloto no publicado.

Fecha de recepción: 30-11-04

Fecha de revisión: 18-05-06

Fecha de aceptación: 17-10-06 